\title{
New Technologies in the Radiotherapy Clinic
}

\author{
J.L. Meyer ${ }^{a} \cdot$ L. Verhey $^{b} \cdot$ P. Xia ${ }^{b} \cdot$ J. Wong

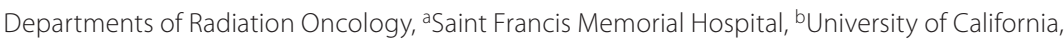 \\ San Francisco, Calif., ' Johns Hopkins University, Baltimore, Md., USA
}

\begin{abstract}
What are the limitations to the accuracy of our current technologies in radiation oncology? The immobilization of the patient, definition of the target, motion of the target and localization of the target are the major concerns that must be addressed. Current approaches to meet these needs have brought new technical systems with greater precision and new clinical procedures with higher expectations of practice. This text offers discussions on these issues, including advances in intensitymodulated radiotherapy planning, clinical target definition for the major tumor sites, management of organ motion, target localization and image guidance systems, and the expanding applications of high-precision treatment with stereotactic body radiotherapy. Copyright $\odot 2007$ S. Karger AG, Basel
\end{abstract}

The technologies of radiotherapy planning and delivery have undergone rapid change. While these changes have been welcomed and carefully nurtured for the benefit of the cancer patient, each change has carried with it a spectrum of new concerns about its appropriate application and efficient integration into radiotherapy practice. These technologies, and the clinical treatment programs that bring them into practical use, are the focus of this volume.

These technical achievements are closely interrelated: one development gives opportunity for another, but often necessitates the creation of a third, and then redefines the use of several others. Understanding this evolving world of new technologies and their applications requires broad perspectives from different vantage points. This volume first takes the viewpoint of computerized treatment planning and delivery with intensity-modulated radiotherapy (IMRT), an elaboration of 
three-dimensional conformal radiotherapy which has been the subject of prior comprehensive volumes in this series. This new level of treatment precision has brought requirements for image confirmation of the targets during treatment and even automated image guidance of the radiotherapy delivery (image-guided radiation therapy, IGRT). It has also brought an exciting new expansion of radiotherapy into the high-precision realm of accelerated stereotactic body radiotherapy (SBRT) for tumor sites outside the cranium. The practical concerns identified in each of these perspectives will be addressed in the sections of this volume.

\section{Intensity-Modulated Radiation Therapy: Where Are We Now, Where Do We Need to Go?}

The intensity modulation of radiation delivery has dramatically changed radiation oncology and greatly expanded the opportunities of the specialty. A little more than a decade ago, IMRT was a new and unconventional idea. Tomotherapy using the Nomos Peacock device was introduced around 1994 and entered use at a few research centers. It was a remarkable innovation, but operationally it carried limiting concerns, including the possible effects that any intratreatment patient motion might have on patient safety or tumor control. By 1996, multileaf collimation had been adapted for IMRT delivery. Its investigation was limited initially to academic centers that were required to develop and maintain appropriate resources in radiation physics not generally available in the community. By the early 2000 s, the acquired experience brought confidence that IMRT could be carried out routinely at comprehensive radiotherapy facilities if the necessary quality assurance programs were provided.

To implement IMRT, the patient-specific quality assurance that must be done is additional but important work. Through these efforts, the number and types of patients benefiting from IMRT have expanded. Also, the time required to perform IMRT has decreased significantly, allowing clinics to treat more of their patients with this approach. Clinical results supporting the use of IMRT now exist for head and neck, prostate and other cancers. In many cases, they show that increasing the dose to the tumor can increase rates of local control while decreasing the dose to normal tissues can reduce complications. The clinical results with IMRT are actually occurring as many predicted they would. Yet the development of more precise means of delivering radiotherapy has brought new concerns, especially regarding patient stabilization, organ movement, tumor tracking, and treatment reproducibility.

What has changed? Most importantly, the efficiency of the clinical operations has changed. The efficiency of IMRT planning and delivery is approaching or even exceeding that of complex three-dimensional conformal therapy. Advancements 
Table 1. Estimated decrease in planning and treatment times since 2002 for complex head and neck IMRT at UCSF

\begin{tabular}{|c|c|}
\hline Contouring time & No change (estimated $2 \mathrm{~h}$ per case) \\
\hline Planning time & $\begin{array}{l}\text { Decrease by a factor of } 2 \text { (from } 4 \text { to } 2 \mathrm{~h} \text { ) due to improved } \\
\text { understanding of appropriate prescription information for } \\
\text { optimization }\end{array}$ \\
\hline Quality assurance ${ }^{1}$ & $\begin{array}{l}\text { Decrease by a factor of } 2 \text { (from } 2 \text { to } 1 \mathrm{~h} \text { ) due to experience, better } \\
\text { equipment }\end{array}$ \\
\hline Treatment time & $\begin{array}{l}\text { Decrease by a factor of } 4 \text { due to better delivery algorithm, better } \\
\text { choice of angles, contour-based inverse planning algorithm } \\
\text { (from } 120 \text { segments } / 40 \text { min to } 50 \text { segments } / 10 \text { min as of later in } 200\end{array}$ \\
\hline
\end{tabular}

${ }^{1}$ Measurements in phantom.

in treatment planning algorithms are now providing delivery of simpler treatments with equivalent quality. Image-guided radiotherapy, in one of its several available forms, offers the expectation that the treatment target can be localized as needed at the time of treatment, to decrease margins and make the dose delivery safer. The developing technologies for four-dimensional CT are now being used for planning and dose modification. Complex image-guided radiotherapy, as with daily megavoltage or kilovoltage cone beam CT imaging in the treatment room, can lead to a volumetric analysis of the actual dose delivered to the patient on a daily basis. It will be challenging to use and integrate all of this available information, called dose-guided radiotherapy, yet it offers a new level of understanding and quality assurance for every treatment delivered in a therapy course. Soon it may be an expected standard of care.

\section{IMRT Efficiency and Benefit}

IMRT planning and delivery can be examined in each of the work phases to show where efficiencies are improving (table 1). At the University of California, San Francisco (UCSF), the time required for contouring has not changed greatly over the past few years; the algorithms for contouring have improved, but the contouring of tissue volumes still requires about $2 \mathrm{~h}$ for an average head and neck case. The planning time itself has decreased by half, from about 4 to $2 \mathrm{~h}$ per case, largely because of a better understanding of what specific prescription information leads to the desired dose distributions for a given patient group. This efficiency can be attributed to the greater experience of the planners more than to the development of the planning systems. Quality assurance measurements require about $1 \mathrm{~h}$ per patient before the first treatment, again about half the time spent earlier. The actual treatment time, a precious commodity in the operation of any clinic, has diminished by almost a factor of four over the past 3 years. This reflects two 

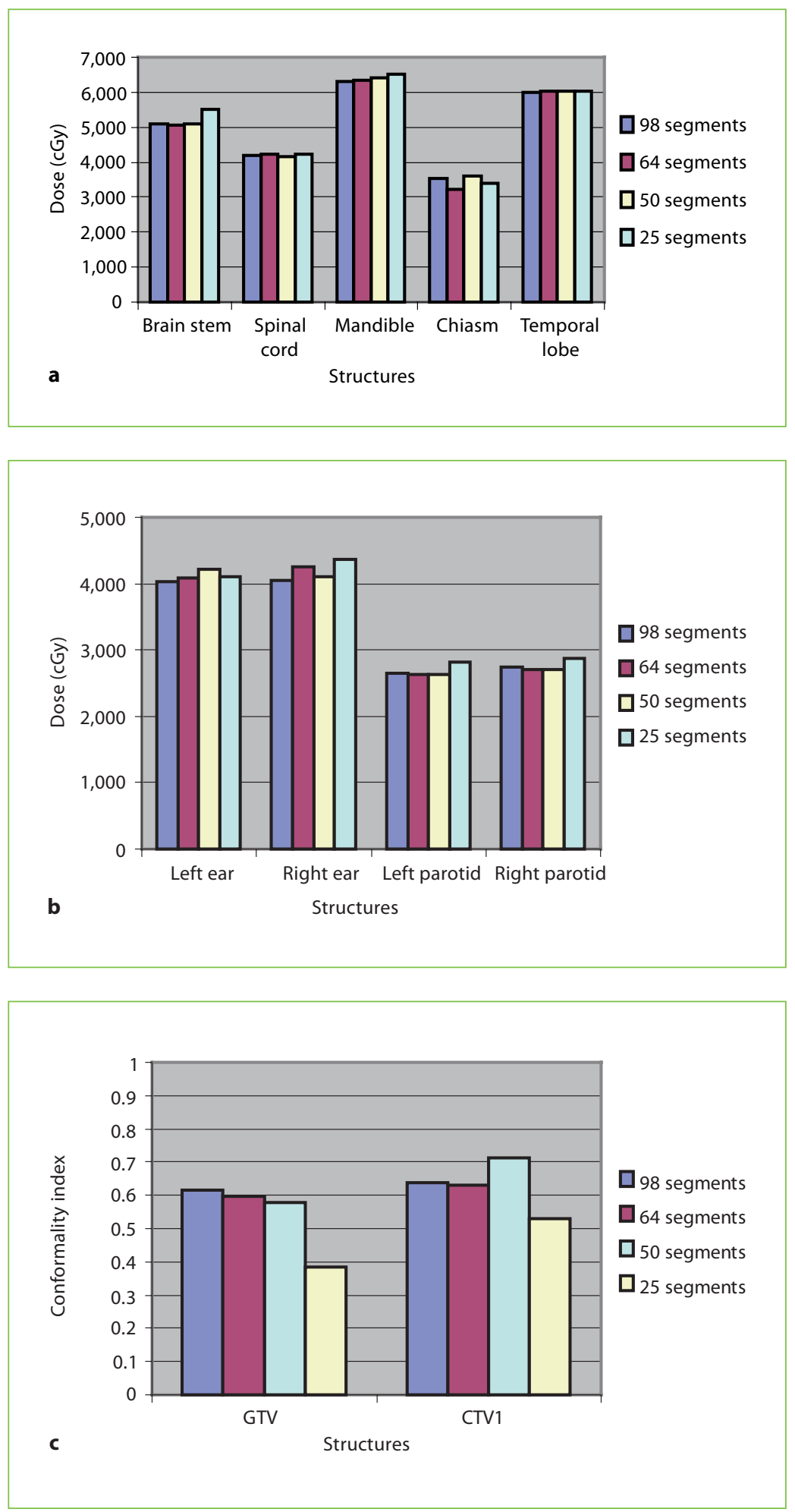


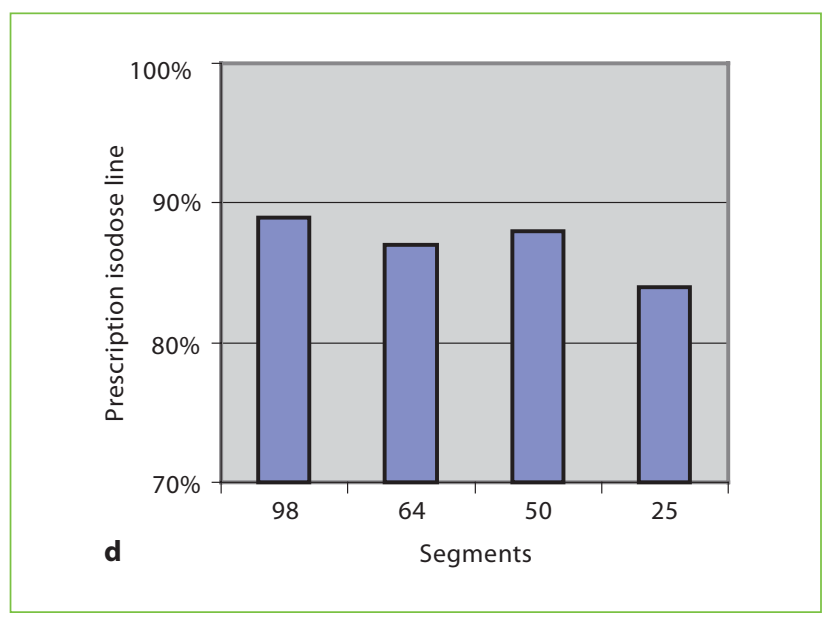

Fig. 1. Effect of the number of segments. Control of dose to normal tissues, conformality and uniformity indexes decrease below 50 segments. a Average maximum dose to $1 \mathrm{cc}$ of serial structures. b Average mean dose for parallel structures. c Average conformality index. d Average uniformity index.

changes. First, the new algorithms for inverse planning that use optimization based on anatomic contours or apertures, instead of pixels, have reduced treatment time by half. Second, the number of treatment segments has been reduced by about $50 \%$ with no appreciable loss of quality. At UCSF, the most complex IMRT cases now use about 50 segments delivered over an average of 7 angles, which require about $10 \mathrm{~min}$ to deliver after the patient is set up. Previously, treatments required $25-35 \mathrm{~min}$, and as long as $40 \mathrm{~min}$ for complex cases that were using 120-150 segments. This improvement is a breakthrough in efficiency, and anticipates a time in the near future when the most complex cases need only $15 \mathrm{~min}$ in the treatment room.

What level of IMRT complexity should be used? The balance between clinical efficiency and therapeutic benefit of IMRT segmentation has been approached in work at UCSF [1]. Their previous experience with pixel-based optimization algorithms showed that for simple head and neck cases, IMRT plans typically required 90 segments with 6-7 beam angles, but for complex head and neck cases as many as 130-160 segments with 9 beam angles were needed. The question is, can plans with equivalent quality be obtained with fewer segments? New planning algorithms have potentially made this possible. Using an aperture-based optimization algorithm implemented in the Pinnacle planning system, the maximum number of segments permitted in the optimized plan can be specified. Figure 1 shows the effect of 98, 64, 50 or 25 segments on several normal tissues, shown as serial or parallel organs. 
As seen particularly for brain stem volumes, it is evident that there is a deterioration of the quality of the plan observed below 50 segments, whereas the results for 50 or more segments are similar. The conformality of the prescription dose line is significantly different at 25 segments than it is at $50-98$. The uniformity index (the prescription isodose line that is used in the prescription) begins at about $89 \%$ for 98 segments, and is about the same for 50 segments. At 25 segments, it has diminished to around $84-85 \%$, which is probably a significant decrease clinically. For the complex treatment plans in this study, the number of segments could be reduced by about half, from around 100 to around 50, without sacrificing quality.

\section{Heterogeneity Correction}

The manner of dose calculation is an essential aspect of accuracy in the planning process. The use of dose heterogeneity corrections for planning throughout the body is now considered standard in most radiation therapy clinics, and is especially important in thoracic sites. There are several different methods used to perform dose heterogeneity corrections, and some can approach (within a few percentage points) the results of exhaustive Monte-Carlo-based calculations. Convolution superposition has become a standard algorithm over the past few years. While the results obtained by these methods can vary, these differences are far smaller than the effects of not using heterogeneity corrections.

\section{Image Guidance in Radiation Therapy}

Tumor and normal tissues move with time, and this movement may be clinically significant from second to second, day to day, week to week, or longer. The movement may be periodic and predictable (like respiratory motion), irregular (like peristalsis), or even permanent (like tumor shrinkage). From a radiotherapy point of view, these variations may be considered intratreatment or intertreatment. In actuality, every tumor site will show both of these effects to varying degrees; some will be dosimetrically significant while others will not. For instance, a lung tumor may move with respiration; show three-dimensional rotational changes from day to day; be affected gradually by changing atelectasis, edema and fluid, and gradually shrink during a therapy course. Even repeated CT scanning will have difficulty in capturing all of these changes in each snapshot of imaging.

Each tumor site (and to some extent, every tumor) will have its own characteristics of movement. For thoracic tumors, periodic respiratory motion often predominates the pattern of change, while for head and neck tumors it is gradual tumor shrinkage over time. Prostate tumors may change position primarily day by day, though additional momentary and irregular changes can occur as a result of peristalsis in a minority of patients. However, no tumor appears to be immune 
from some combination of all of these momentary and more gradual changes, some of which may be complex and unpredictable. For all of these differences, how can the delivery of uniform radiotherapy dose to the targeted tissues be guaranteed? To embark on this journey, work in radiotherapy has begun to tame periodic motion through restriction of motion (e.g. breath hold, with or without assistance), prediction of motion (e.g. gating, four-dimensional CT reconstructions), or tracking of motion through robotics or other dynamic approaches, and will be discussed in this volume.

\section{Intertreatment Changes}

At present, radiation oncologists have the greatest opportunity to immediately improve therapy delivery through the identification and correction of change occurring between therapy fractions. Intertreatment motion can be studied by imaging the patient using megavoltage or kilovoltage cone beam systems referenced to the planning system or by other approaches. The imaging can be obtained on a regular, predefined basis or at specific points during a course of therapy.

Evaluation of intertreatment change is important in several areas, especially the head and neck region. Figure 2 projects the IMRT dose distributions for a head and neck cancer patient treated at UCSF; 70 Gy is planned to the gross tumor volume (GTV) and 59.4 Gy to the clinical target volume. In figure 2a, the treatment plan based on the initial CT is shown. After 21 treatment fractions were given, the tumor had markedly regressed and the patient had lost $5 \%$ of his body weight. A second CT was obtained, and figure $2 \mathrm{~b}$ shows that the original plan now projects differently on the tissue structures, since some of the volumes have changed. For instance, the dose to the spinal cord is much higher than intended. Figure $2 \mathrm{c}$ shows the reoptimized plan based on the second CT.

Similar work was performed in a series of patients at UCSF; CT studies were repeated in head and neck cancer patients if their contour had noticeably changed, which is fairly common in this patient group [2]. The two CT studies were typically $4-5$ weeks apart. For each case, a recalculation of the doses was performed, with endpoints being the dose to $95 \%$ of the target volumes, the maximum doses to the spinal cord and brain stem, the mean doses to parallel structures (mainly the parotid glands), and the total doses. Analysis of the normal and tumor tissue volumes indicates that the doses to the right and left parotid glands were reduced by 15.6 and $21.5 \%$, respectively, and the clinical target volume dose decreased by $7.5 \%$. Figure 3a illustrates the dose to $95 \%$ of the GTV that would have been delivered with or without replanning. Substantially lower doses would have been delivered to the GTV than intended by the initial plan in most cases. Figure $3 b$ shows the spinal cord doses predicted by three plans for each case: an initial plan and a second reoptimized plan, and a third showing the first intensity patterns applied 

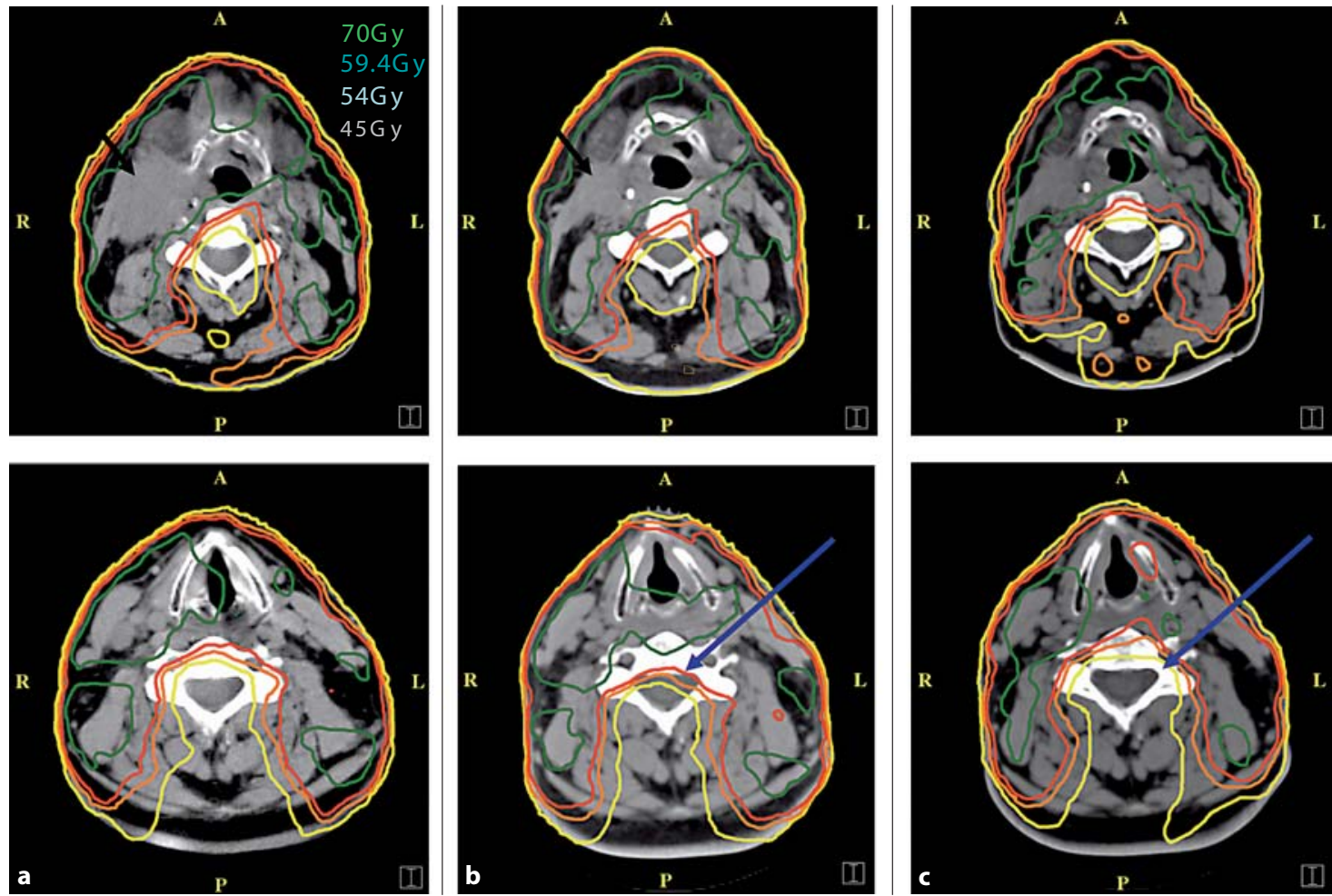

Fig. 2. IMRT treatment isodoses for a T4N2c base of tongue cancer in a 54-year-old male. Top row: level of hyoid bone; bottom row: lower neck. a CT 1: initial plan, before therapy. $\mathbf{b}$ CT 2: same plan shown on repeat CT scan after 21 fractions; tumor has regressed (black arrow) and patient has lost $5 \%$ of his body weight. Note that spinal cord dose has become unacceptable (blue arrow). c CT 2: reoptimized plan.

to the second CT scan. If replanning and reoptimization were not performed, significantly higher doses might have been given to the spinal cord than were intended. It is important to follow the patient and perform replanning when needed, and detection of significant soft tissue changes may be one of the most useful applications of image guidance with cone beam technology.

\section{Intratreatment Motion and Tumor Tracking}

The issues involved in imaging, tracking and managing motion during the treatment itself are challenging, though they may ultimately provide the best answers to treatment verification. Platforms now exist for three-dimensional radiographic tracking of passive implanted fiducial seeds and the radiofrequency tracking of implanted interactive seeds. Flat-panel technologies used in cone beam CT offer the potential for fluoroscopic monitoring of anatomy or fiducials at kilovoltage or 

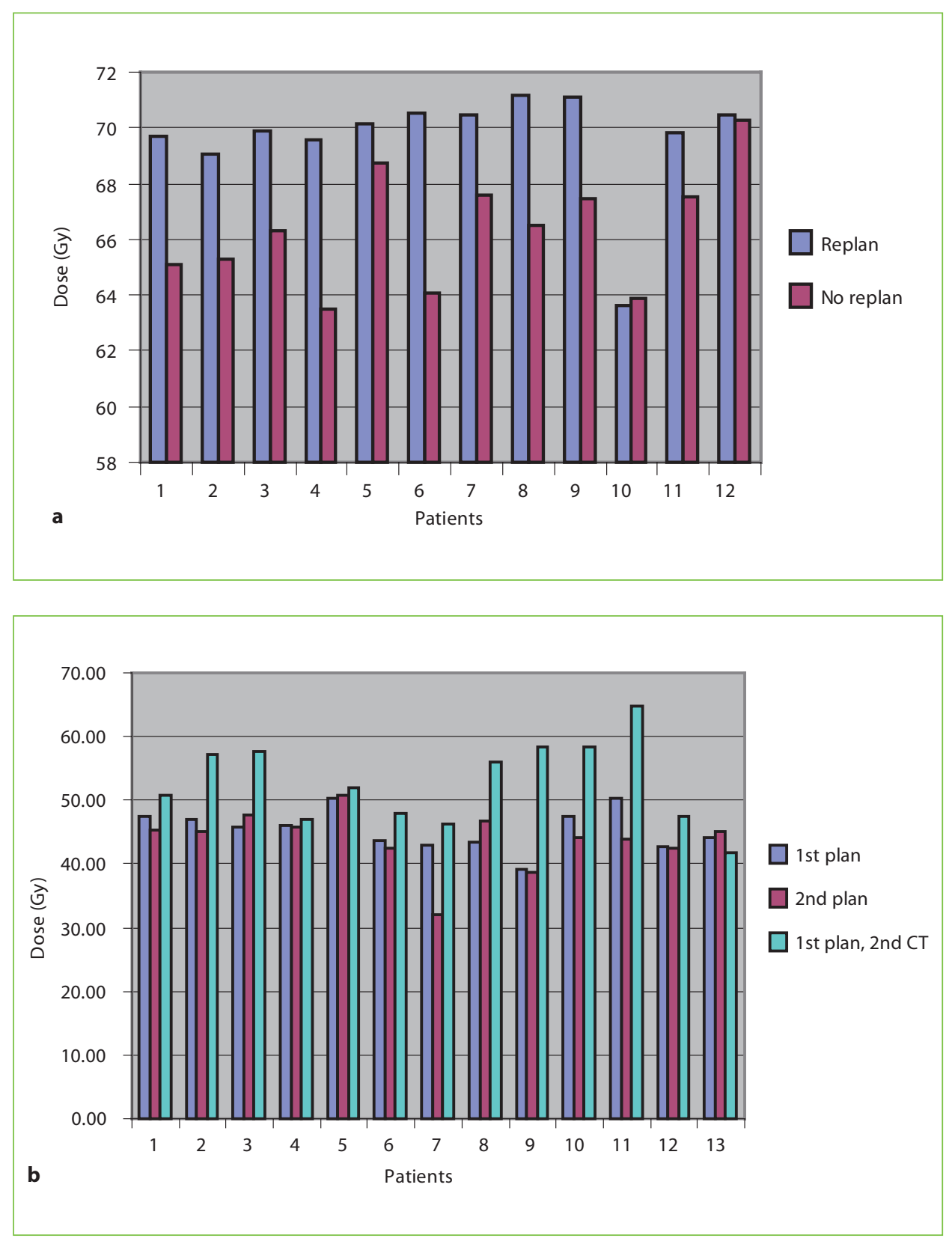

Fig. 3. Effect of replanning on head and neck cancer cases. a Dose to $95 \%$ of GTV (D95). Deterioration of tumor dose without replanning. b Maximum dose to spinal cord. Initial plan and replan doses to normal structures. Third bar shows doses to normal structures if replanning was not performed. Note potentially excessive doses to critical normal structures. 


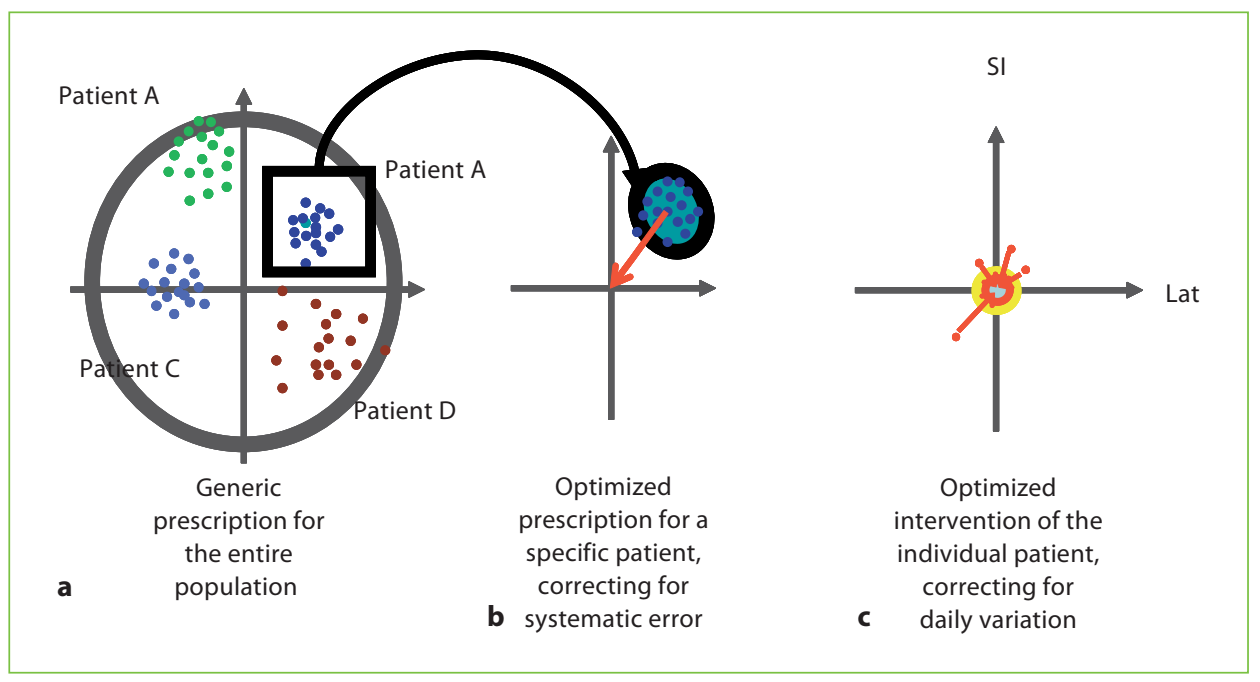

Fig. 4. ART strategies for correcting target positional variations. a Conventional radiotherapy, enlarging PTV for all patient variations. b ART, correction for systematic variations in a treatment course. c ART, correction for all systematic and random daily variations. Circle and elipse show required PTV expansion.

megavoltage energies. Algorithms are now being developed for on-line automated monitoring of targets or fiducials using cone beam technologies. These may even integrate a computer-directed decision about when to stop a treatment and make adjustments in the occasional cases that may require this. The quality of kilovoltage and megavoltage cone beam images is already sufficient for these processes, and the transfer of these technologies into the clinical setting in commercial products is a logical next step. This may include an evolution of the technologies to permit tracking of close tumor surrogates, perhaps adjacent bone contours, rather than implanted markers so that these may not be required in every case.

\section{Adaptive Radiotherapy}

Modern radiotherapy is all about defining the target - for clinical tumor delineation, treatment planning, and radiation delivery to the planning target volume (PTV). As new biologic methods and refinements of traditional imaging tools give better tumor delineations, and as greater efficiency and accuracy of treatment planning processes develop, the accurate delivery of the daily treatment itself emerges as a foremost concern. Imaging in the treatment room has provided extensive data documenting movement and anatomic change of targets during and between therapy sessions that can be clinically significant. We are challenged to represent, control and/or incorporate these changes in strategies to manage target movement and setup uncertainty. 
The ability to develop automated mechanisms to identify and correct such treatment variations is a major objective, called adaptive radiation therapy (ART). Introduced by the William Beaumont Hospital group in 1997 [3], the concept of ART includes the following key features: it incorporates systematic measurements of treatment variations into a closed-loop radiation treatment process, provides feedback to reoptimize the treatment plan early on during the course of treatment, and delivers treatment that is customized to the daily patient target volumes.

The goals of ART are illustrated in figure 4 . In figure $4 \mathrm{a}$, the clusters of dots represent the actual daily setup positions for 4 patient treatment courses. In an ideal course, the patient would always be set up at the prescribed isocenter (the interception of the $\mathrm{x}$ - and $\mathrm{y}$-axes in the figure). In practice, the actual daily setup positions are found to be distributed at distances away from this ideal position. If one wants to adjust the PTV expansion margins to accommodate for all such variations found for an entire treatment group (for instance, all prostate cancers treated with IMRT), then a quite large treatment margin would need to be added (represented by the shaded circle). Avoiding such large expansions requires on-treatment imaging of the individual patient, and is the reason for the image guidance of radiotherapy. This imagining information can reveal two types of variation that may be occurring: systematic, in which the mean of the observed positions is offset by a measured xyz amount from the prescribed position, or random, which can be measured as daily changes from this mean.

The systematic type of uncertainty is amenable to off-line analyses of imaging acquired at treatment. In figure $4 \mathrm{~b}$, patient $\mathrm{A}$ can be effectively treated with a smaller margin if the systematic error is identified early on during the treatment course and corrected, as demonstrated by the shift with the arrow. Now a smaller margin can be prescribed, since it only needs to account for the random variations specific to the patient. In general, a systematic error is the more detrimental, because the dose is being consistently delivered to an unintended location. Random errors are more forgiving, since any effects on the final dose distribution tend to smooth out during the treatment course, especially if it involves many fractions. However, when a treatment course involves high doses delivered using a small number of fractions, large random variations become unacceptable. Random errors can only be addressed with daily on-line imaging and modification. Figure 4c illustrates the example of a treatment course where every daily variation is observed and corrected, permitting the smallest PTV margins to be used.

It is estimated that the majority of treatment errors can be corrected through off-line analyses. For example, figure 5 shows the results for 300 prostate cancer patients that were treated at William Beaumont Hospital using an ART protocol. On-treatment imaging was obtained for each patient, the mean setup variation over the first week of therapy was determined, and an adjustment for any systematic error was made off-line. The figure shows that about $80 \%$ of treatments would 


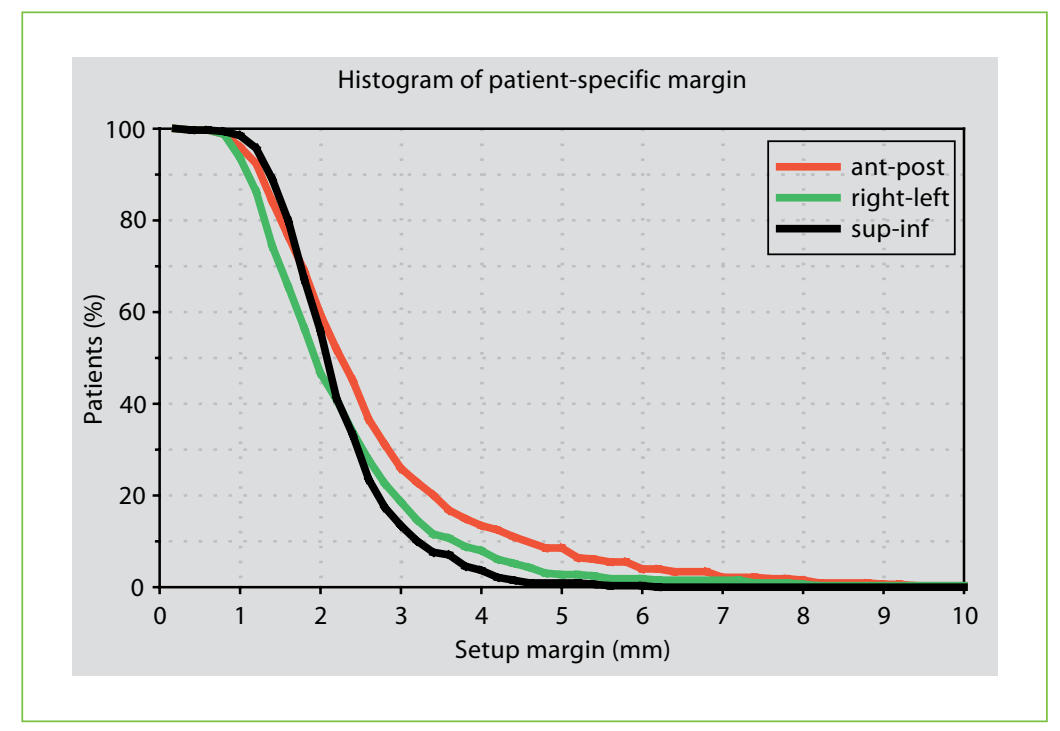

Fig. 5. ART protocol for prostate cancer cases (see text). After off-line correction of systematic error, about $80 \%$ of setup variations can be brought within $3 \mathrm{~mm}$ of the planned isocenter, and about $20 \%$ will gain from further on-line guidance. The gain in setup margin reduction is substantial and can be readily achieved by the general clinic.

then be within $3 \mathrm{~mm}$ of accuracy, and could be accurately accounted for by a PTV expansion of $3 \mathrm{~mm}$ or less. In only $20 \%$ of cases would daily on-line guidance be needed to bring treatments within this expansion margin.

The ART mechanism must identify setup errors and determine whether they represent systematic variation. If there is certainty of a change, then ART must drive a correction process, making the delivery system understand the referenced location of the isocenter. In the case of random setup differences, their magnitude must be identified in order to provide exact daily correction. Alternatively, ART may drive a change in the planning volume encompassing these daily variations or the delivery technique itself to reduce them. Ultimately, ART involves recognizing the daily treatment delivery process and adjusting it as required by identified positional or volumetric variations of the target.

To achieve these goals, ART integrates replanning and dose reconstruction tools. It requires infrastructure for distributing information and tools for off-line decision making to approve or disapprove actions. New skills must be learned to make better use of these new technologies, and the radiation treatment process must be redeveloped as an informatics system for the efficient management of imaging data for treatment control. 
ART is patient, tumor site, equipment and institution specific. Practical development of ART requires an understanding of how the PTVs were developed for the individual patient or for a patient population as a class solution. These standards will be specific to each institution, which must then address fundamental questions. What should be the limits for on-line image-guided setup correction? How will these vary when imaging is directed by soft tissue contours, implanted markers or other surrogates? How reproducible is the clinic's management of breathing motion by gating or other strategies? What is the appropriate PTV after the introduction of a new IMRT method, which may be different than the PTV used before this technology? These decisions must be part of a directed process within the clinic's self-assessments, and an effective ART method will depend on having a well-established and continuing process of radiotherapy quality assurance overall.

\section{Biology, Imaging and Image Guidance}

Practical issues now confront the current treatment planner, whether physician, physicist or dosimetrist, that can only be answered in the realm of further biologic investigation. If one identifies shrinkage of a tumor during radiotherapy, what should be done? If a tumor appears to increase and edema is suspected, should the target be increased? How does a physical anatomic change in the tumor volume reflect a biologic functional change that permits a target change? As yet, few biological or clinical studies have addressed this issue. PET might be used; however, the significance of changes in its standard uptake value units is not understood, and indeed the tumor/blood ratios involved with this calculation may be altered during radiotherapy. Also, the measurement of the tumor size may vary based on the resolution or intensity adjustments used in the PET scan procedures. Perhaps molecular markers could be of help by allowing functional tracking of treatment-induced changes; however, biological and clinical evaluations are still needed to create useable clinical systems. At present, it is difficult to know how weekly radiographic changes in tumor volume reflect biologic changes. Many investigators feel that there is little scientific basis for modifying therapy volumes based on identified tumor changes during treatment, though treatment replanning may be important to correct for contour or soft tissue changes so that the originally intended dose distributions are achieved.

\section{Stereotaxis and Radiotherapy Delivery}

The field of stereotactic radiosurgery is not new, and is based on work by pioneering Swedish investigators led by L. Leksell (1907-1986). More than 50 years ago, he investigated combining stereotactic surgery techniques with external beam 
irradiation [4]. In 1956, about the same time that similar work began in the USA, Larsson and Leksell initiated proton radiosurgery in Uppsala, Sweden [5]. Some 12 years later, these two individuals led the development of Gammaknife cranial radiosurgery, and in 1972 Leksell and his son founded Elekta Instruments to manufacture this and other related devices. A linear accelerator alternative to gamma irradiation was described in 1983 [6]. Now cranial radiosurgery has become a standard of care for benign and malignant brain tumors of all histologies, and for numerous benign conditions including arteriovenous fistulas, trigeminal neuralgia and other pain conditions, and movement disorders including epilepsy and Parkinson's disease.

Based on the decades of experience with brain stereotaxis, investigators extended work to extracranial treatment using linear accelerator delivery systems. Workers at the Karolinska Institute, Stockholm, Sweden, presented a report on the stereotactic therapy of abdominal tumors in 1994 [7]. A body immobilization frame for thoracic radiosurgery was developed, which reported reproducibility within $5-8 \mathrm{~mm}$ for $90 \%$ of setups. At the same time, diaphragmatic movement could be limited to 5-10 $\mathrm{mm}$ with the use of an abdominal pressure device. The following year, an original clinical report described stereotactic radiotherapy of 31 patients with primarily solitary tumors in the liver and lung who received doses of 7.7-45 Gy in 1-4 fractions [8]. In the USA, spinal radiosurgery was reported in 1995 [9].

At the same time, essential work in SBRT was undertaken at the National Defense Medical College, Japan. Beginning in October 1994, 45 patients with primary or metastatic lung cancers were treated using a linear accelerator with in-room CT guidance. Radiation doses were 30-75 Gy in 5-15 fractions over 1-3 weeks, with or without conventional radiation therapy [10]. Careful protocol work commenced soon thereafter in the USA and is well reported in this volume.

The first SBRT investigations occurred during the same years that IMRT was introduced, and these concepts were logically married in new planning and delivery systems. In fact, the Cyberknife device was first conceived by J. Adler during work at the Karolinska Institute in the late 1980s, and practical development of the system began in 1990. Combining stereotaxis with image guidance and computercontrolled robotics, the Cyberknife was first cleared for use in the USA beginning in 1999. Extracranial treatments currently represent more than $50 \%$ of the procedures performed, including those for spine, lung, prostate, liver and pancreas sites. Currently, there are more than 100 Cyberknife centers in the USA, Japan, Europe and other areas of the world.

Concurrently, similar work developed a linear accelerator-based system for extracranial stereotactic radiosurgery using micromultileaf collimation. Founded in 1989, BrainLab developed commercial systems first available in 1993. Current- 
ly, the BrainLab Novalis system is open or planned for service in nearly 100 facilities worldwide. Since these initial specialized equipment developments, nearly all other manufacturers of radiation treatment systems have introduced features well-suited or easily adaptable for SBRT.

\section{Current Concerns for SBRT}

The technical progress in image-guided IMRT and dedicated radiosurgical delivery systems have assisted in the planning and delivery of SBRT, though effective SBRT work was performed prior to their introduction, and the safe use of this high-dose treatment method depends primarily on the advanced training of its personnel and their careful management of patients. While equipment is still developing for the accurate delivery of stereotactic radiosurgery for tumors outside the cranium, many fundamental biological and clinical questions remain regarding the use of these technologies in medical practice.

\section{Dose}

The cell survival curves that serve as the basis for conventional radiotherapy practice are derived from older laboratory studies characterizing in vitro cell survivals to single-fraction treatment over the first 2-3 logs of cell kill. These analyses fall short of the level of cell kill caused by the high fractions of SBRT, and the shapes of survival curves at higher fraction levels are not well understood. Therefore, the mathematical modeling of these curves, including their power series expansion coefficients, are not dependable either. Without this accurate modeling, it becomes difficult to extrapolate between the anticipated results of one fractionation scheme and another, and difficult to know whether any given treatment course may result in relative overtreatment or undertreatment in terms of tumor control or normal tissue preservation. What is known, in terms of the alpha/beta model that is currently understood, is that this model likely overpredicts the effectiveness of large-dose SBRT treatments.

\section{Fractionation}

Original investigations in Sweden used, on average, 3 treatment fractions for SBRT. While this selection may have merit, neither clinical nor biologic investigations have secured clear evidence for this choice. Since multiple treatments have been used, some element of biological effect has been recognized in these protocols. It is likely that this effect could be optimized through more careful analysis, giving physicians a clearer basis for their treatment specifications. From conventional radiobiology, it is probable that the host site of the tumor, whether liver, lung, bone or other tissue, will have different tolerance to the fraction size and total dose, as well as the field volume. This evidence can only be obtained through scientific studies using consistent treatment approaches and monitoring well-defined endpoints. 


\section{Patient Selection}

There is no reason to suppose that all patients or tissue organs will tolerate SBRT equally well. From the science of radiotherapy, it is understood that toxicities to large radiation fractions are predominantly late occurrences. Though rare, these have been observed after SBRT in the bronchus, bowel, spinal cord, and other organs. Since these toxicities may occur late, longer follow-up will be required for wider acceptance of SBRT. At the same time, it is imperative that careful work be performed on the selection of appropriate candidates for therapy to establish a broader range of tumor-specific applications. Considerations may include the prior radiation history of the treatment tissues, treatment volume, organ function and capacity for recovery. Similarly, there is no reason to suppose that all patients will benefit equally from SBRT. Selection criteria might include the number of sites of disease, prior systemic therapy, histology, and many other individual cancer-related factors. Only through a consistent and monitored approach will optimal groups be identified for cancer treatment.

\section{Treatment Availability}

Finally, where will SBRT be delivered? At present, only a small number of radiotherapy centers offer this approach. Is this appropriate, or should it become widely available quickly, as IMRT has become? One aspect of SBRT to consider is the short duration of the treatment courses. This may be one of its chief advantages; for instance, as an alternative for prostate cancer therapy. In fact, the availability of SBRT at facilities offering service to more remote areas may be desirable, since lengthy stays away from home could be minimized. At the same time, it is essential to have a facility offering a high level of professional expertise and technical support. One must consider that the doses delivered by SBRT are the most highly accelerated and focused of any in radiation treatment, and may have the greatest potential for radiation injury. SBRT clearly requires management by radiation specialists well-trained and certified in its exacting requirements.

\section{References}

1 Ludlum E, Akazawa C, Xia P: IMRT plans can be simplified using one step optimization. Med Phys 2006;33:2111

2 Hansen EK, Bucci MK, Quivey JM, Weinberg V, Xia P: Repeat CT imaging and replanning during the course of IMRT for head-and-neck cancer. Int J Radiat Oncol Biol Phys 2006;64:355-362.

3 Yan D, Vicini F, Wong J, Martinez A: Adaptive radiation therapy. Phys Med Biol 1997;42:123132.

4 Leksell L: The stereotaxic method and radiosurgery of the brain. Acta Chir Scand 1951:102:316319. 
5 Larsson G, Leksell L, Rexed B, Sourander P, Mair $\mathrm{W}$, Andersson B: The high energy proton beam as a neurosurgical tool. Nature 1958;182:12221223.

6 Betti O, Derechinsky V: Multiple-beam stereotaxic irradiation. Neurochirurgie 1983;29:295298.

7 Lax I, Blomgren H, Naslund I, Svanstrom R: Stereotactic radiotherapy of malignancies in the abdomen. Methodological aspects. Acta Oncol 1994:33:677-683.

8 Blomgren H, Lax I, Naslund I, Svanstrom R: Stereotactic high dose fraction radiation therapy of extracranial tumors using an accelerator. Clinical experience of the first thirty-one patients. Acta Oncol 1995;34:861-870.

9 Hamilton A, Lulu B, Fosmire H, Stea B, Cassady $\mathrm{J}$ : Preliminary clinical experience with linear accelerator-based spinal stereotactic radiosurgery. Technique and application. Neurosurgery 1995; 36:311-319.
10 Uematsu M, Shioda A, Tahara K, Fukui T, Yamamoto F, Tsumatori G, Ozeki Y, Aoki T, Watanabe M, Kusano S: Focal, high dose, and fractionated modified stereotactic radiation therapy for lung carcinoma patients: a preliminary experience. Cancer 1998;82:1062-1070.

Dr. John L. Meyer

Department of Radiation Oncology

Saint Francis Memorial Hospital

900 Hyde Street

San Francisco, CA 94109 (USA)

Tel. +1 415353 6420, Fax +1 4153536428

E-Mail JMeyerSF@aol.com 